\title{
La progression des enseignements-apprentissagesdu Français langue première au secondaire: une proposition didactique
}

\author{
Suzanne Chartrand \\ Université Laval \\ Suzanne.Chartrand@,fse.ulaval.ca
}

\begin{abstract}
Depuis la fin du $\mathrm{XIX}^{\mathrm{e}}$ siècle, l'idée de progression des enseignements-apprentissages scolaires a semblé relever de la nécessité - on ne peut tout enseigner en même temps - et du bon sens - on ne peut organiser un enseignement sans tenir compte du niveau des élèves (Bronckart \& Chiss, 2002). Déjà, Comenius, dans sa Didactica magna (1638/2002), considérait que les programmes scolaires devaient sélectionner et organiser les objets d'enseignement en une progression qui tienne compte des capacités cognitives des élèves. Cela dit, la progression ne semble plus aller de soi, du moins dans les récents programmes d'études québécois pour l'enseignement du français, qui ne présentent pas de progression claire par année ou par cycle des genres de textes à travailler ni des contenus d'enseignement-apprentissage, en particulier ceux relatifs à langue. La notion a peu suscité de débats au sein de la communauté des chercheurs en didactique du français langue première, et s'il fut un temps où il était central en didactique des langues étrangères et secondes, Coste (2000, p. 11) remarque qu'à la fin des années 90 , il ne semble pas faire partie des notions clés de la didactique des langues.
\end{abstract}

Une des tâches du didacticien consiste pourtant à faire l'analyse critique des objets d'enseignement prescrits ${ }^{1}$ et à proposer une progression argumentée de leur enseignement-apprentissage à partir de critères explicites (Aeby, De Pietro et Wirthner, 2001; Coste \& Véronique, 2000), car la progression est un "problème typiquement didactique» (Chiss, 2000: 67) ${ }^{2}$. Notre proposition se situe donc dans la problématique de la dimension praxéologique de la didactique du français. En suivant la tradition des pays francophones, les prescriptions et les pratiques actuelles dans l'enseignement du français en milieu francophone (celles attestées par les moyens d'enseignement, notamment), mais aussi en tenant compte des besoins scolaires et plus largement personnels et sociaux des élèves, et de la complexité de certains genres par rapport à d'autres, nous proposons une progression des objets d'enseignement-apprentissage pour la classe de français du secondaire (de la $7^{\mathrm{e}}$ à la $11^{\mathrm{e}}$ année de scolarisation $\left.{ }^{3}\right)$.

\section{Cadre conceptuel}

\subsection{La notion de progression}

On peut définir la progression des enseignements comme leur organisation en un itinéraire temporel permettant un apprentissage optimum; elle vise donc à régler la chronogénèse. La planification « rationalisée et économique » (Chiss, 2000: 70) des objets d'enseignement-apprentissage relève du paradigme pédagogique selon lequel le développement langagier et cognitif optimum des sujets requiert un enseignement formel explicite des connaissances, des habiletés et des attitudes constitutives des disciplines scolaires. Comme le revendique Chiss (2000: 70), si la progression doit privilégier un point d'ancrage, c'est le «savoir de l'enseignant », car il est nécessaire « d'avoir déjà construit pour construire avec les élèves ». 
Diverses progressions sont utilisées en éducation: la progression linéaire (du plus simple au plus complexe; du plus fréquent au plus rare; du général au spécifique), la progression par accumulation (chaque objet est découpé en parties) et la progression spiralaire (par approfondissements successifs), que nous privilégions. Elle permet d'intégrer les autres progressions pour des apprentissages spécifiques bien délimités; elle permet surtout de tenir compte non seulement des contenus à enseigner mais aussi de divers autres facteurs. Elle implique des apprentissages de plus en plus exigeants, car de niveaux de complexité croissante à différents moments du développement cognitif et langagier des élèves (Dolz et Schneuwly, 1996, Bailly, 2000, GREF, 2002, Dolz, Noverraz \& Schneuwly, 2001). Il ne s'agit pas d'un simple rebrassage cyclique, où on enseigne de la même façon et selon les mêmes objectifs le même phénomène trois fois de suite ou pendant trois cycles consécutifs (l'accord de l'adjectif ou du verbe, notamment, qui est travaillé sensiblement de la même façon du primaire à la fin du secondaire). Faut-il rappeler, à l'instar du GREF (2002: 3), que la maitrise de la compréhension et de l'expression est illusoire « sans un travail rigoureux, structuré, soutenu dans la durée par de bons outils $»$ ?

\subsection{Les critères d'établissement d'une progression}

Pendant longtemps, la progression des enseignements-apprentissages de la langue dans le cadre de la classe de français langue première ${ }^{4}$ a été établie à partir du critère de la complexité du phénomène grammatical à enseigner, même si le phénomène, par exemple la phrase subordonnée relative, comprend des réalisations de complexité variable. Nous proposons plutôt d'organiser la progression selon un faisceau de critères:

1. Le niveau de développement cognitif et langagier des élèves, entre autres leur capacité à abstraire et à conceptualiser, de même que leur compréhension des catégories sémantiques (celles de temps et d'espace sont maitrisées plus tôt que celles de cause et de concession...). Dans ce domaine, on ne possède pas encore toutes les données psycholinguistiques utiles, aussi doit-on adopter un empirisme nourri par les recherches en didactique des langues et la connaissance des milieux scolaires. Chose certaine, la réflexion actuelle rompt avec les représentations simplistes, « images de linéarité, de continuité et d'homogénéité » des apprentissages (Coste, 2000:11).

2. Le phénomène grammatical considéré ${ }^{5}$ en tenant compte des éléments suivants:

- la difficulté des élèves à maitriser le phénomène en situation de communication;

- le statut du phénomène dans la langue (le nom et le verbe sont non seulement des mots très fréquents, mais ils ont aussi un statut plus important que l'adverbe, par exemple; noyaux du groupe nominal et du groupe verbal, ce sont les constituants de $1^{\text {er }}$ niveau de la phrase);

- la complexité du phénomène dans le système de la langue ou dans le langage (les relatifs complexes et les dérivés de lequel par rapport aux relatifs qui et que);

- la fréquence du phénomène (les subordonnées compléments de phrase de temps et de lieu sont plus fréquentes que celles de concession; la virgule, plus employée que le point-virgule).

3. L'articulation entre l'étude de la langue et sa réalisation dans les textes lus, étudiés et produits à l'oral comme à l'écrit. Il s'agit de travail dans la langue, pas seulement sur la langue (Bailly, 2000).

4. Les attentes sociales et les prescriptions scolaires, de même que la tradition des différents systèmes scolaires et les cultures enseignantes. Boutan (2000) montre bien que l'idée moderne de progression s'est imposée à cause d'impératifs scolaires et sociaux de la fin du XIX ${ }^{\mathrm{e}}$ siècle en France. 


\subsection{Les phases d'apprentissage}

$\mathrm{Au}$ secondaire, en continuité avec le primaire, la progression peut comprendre trois niveaux d'objectifs d'enseignement-apprentissage. Première phase: sensibilisation. L'enseignant attire l'attention des élèves sur un phénomène grammatical à partir de réalisations représentatives du langage oral et écrit, y compris celui des élèves; il en fait observer la fréquence et l'intérêt discursif (apport au texte, effet produit sur le lecteur); il soulève des questions sur ses caractéristiques sémantiques, morphologiques et syntaxiques; il fait voir la nécessité d'en mieux comprendre le rôle et le fonctionnement, et d'en saisir l'intérêt dans la communication, afin de mieux le maitriser. Deuxième phase: enseignement-apprentissage systématique et approfondi du phénomène grammatical, réalisé à long terme (jusqu'à plusieurs semaines) au moyen de dispositifs didactiques ${ }^{6}$. Comme tous les élèves n'atteignent pas en même temps une connaissance et une maitrise acceptables, une troisième phase est souvent nécessaire. Troisième phase: consolidation des apprentissages ou leur poursuite ou leur approfondissement. Cette phase n'est pas nécessaire si l'étude systématique dans un temps limité suffit, par exemple pour l'apprentissage de conjugaisons ou de structures de phrases plus facilement maitrisables.

Établir une progression articulant développement des capacités langagières (lire, écrire, parler) et connaissance et maitrise de la langue rencontre une difficulté particulière: maintenir l'équilibre entre la nécessité d'une programmation et le besoin de souplesse pour faire face aux contextes didactiques. C'est conscient de cette tension que nous avons conçu cette progression en liaison avec l'étude de divers genres textuels didactisés.

\section{La progression des objets d'enseignement-apprentissage pour la classe de français du secondaire}

Chaque tradition scolaire a établi une progression des textes à travailler en classe à partir d'une typologie? Par exemple, au Québec, le programme de 1980 prescrivait l'étude des discours narratif, expressif, analytique, argumentatif, poétique, etc.; celui de 1995 (MEQ, 1995) imposait l'étude d'un certain nombre de textes répartis selon différents types, à partir de la typologie des séquences textuelles narrative, descriptive, explicative, argumentative et dialogale de J.- M. Adam $(1992 / 2005)^{8}$. L'utilité d'appuyer l'enseignement du français sur une typologie est un acquis de la didactique du français des vingt dernières années. Selon Schneuwly (2002, 1994), la recherche a montré que les élèves comprennent mieux ce qu'ils lisent et produisent des textes plus conformes aux normes et aux règles de l'univers discursif s'ils ont été mis en contact avec les régularités des genres qui agissent comme des repères (De Pietro \& Schneuwly, 2003; Dolz \& Schneuwly, 1998). Le genre est « objet et outil de travail pour le développement du langage » (Schneuwly \& Dolz, 1997 : 33). Aussi avons-nous retenu le principe typologique du genre plutôt que celui du type ou de l'intention de communication.

Bakhtine (1984) a montré que malgré leur apparente diversité, les textes peuvent être regroupés en un nombre relativement limité de genres (premiers et seconds) facilement reconnaissables par les membres d'une communauté linguisticoculturelle, le genre étant défini comme une forme conventionnelle relativement stabilisée présentant des caractéristiques discursives, textuelles et linguistiques spécifiques. Schneuwly (1995) en fait un méga-outil sémiotique dans la conquête de la communication langagière. L'entrée dans l'univers foisonnant de la communication langagière par l'étude de textes d'un même genre, par contraste avec d'autres genres, donne à l'élève des repères essentiels, en compréhension et en production de textes, sur leur but, l'univers représenté, leur structuration, leur mode de mise en texte dominant (narration, description...) et leurs caractéristiques langagières. "C'est au travers des genres que les pratiques langagières s'incarnent dans les activités des apprenants » (Schneuwly \& Dolz, 1997 :29). 
Les textes que lisent les élèves (hormis ceux produits spécifiquement pour l'enseignement d'un phénomène textuel en classe de français), sont d'un genre particulier où dominent une ou des séquences d'un type particulier: un conte ou un roman (séquence narrative), un article encyclopédique ou un horaire de télévision (séquence descriptive), un chapitre de manuel scolaire ou un article de vulgarisation scientifique (dominante descriptive où on trouve des séquences explicatives), un texte d'opinion ou un message publicitaire (séquence argumentative), une pièce de théâtre (séquence dialogale) ou une résolution de problème (genre où domine la justification ${ }^{9}$ ). Cependant, la très grande majorité des textes ont, comme l'a montré Adam (1992/2005), une structure compositionnelle hétérogène. Par exemple, La Peste d'Albert Camus est un roman, donc un genre narratif, où s'enchâssent des séquences dialogales, descriptives, argumentatives et même explicatives.

Dans cette communication, nous exposerons une progression des enseignement-apprentissages de genres classés selon leur structure compositionnelle dominante, susceptibles d'être travaillés en liaison avec les phénomènes discursifs, textuels, lexicaux et syntaxiques les plus pertinents pour un travail systématique et approfondi $\left(2^{\mathrm{e}}\right.$ phase $) .{ }^{10}$ ?

\section{Références bibliographiques}

Aeby, S., De Pietro, J.-F. \& Wirthner, M. (2001). Français 2000. Propositions, interrogations de la recherche pour l'avenir de l'enseignement du français en Suisse romande. Neuchâtel: INRP.

Adam, J.-M. (1992/2005, $2^{\mathrm{e}}$ éd.). Les textes: types et prototypes. Paris: Nathan.

BAilly, D. (2000). Fallait-il vraiment tuer la progression en classe de L2? Dans D. Coste et D. Véronique (éd.). La notion de progression. (pp. 119-132). Saint-Cloud: ENS Éditions.

BaKhtine, M. (trad.1984). Esthétique de la création verbale. Paris: Gallimard.

Béguelin, M.-J. (coor., 2000). De la phrase aux énoncés: grammaire scolaire et descriptions linguistiques. Bruxelles: De Boek/Duculot.

Besson, M.-J., Genoud, M.-R., Lipp, B. \& Nussbaum, R. (1979). Maitrise du français. Méthodologie pour l'enseignement primaire. Neuchâtel: IRDP.

Bilodeau, S. (2005). Le décloisonnement des activités de la classe de français. Analyse d'écrits en didactique du français [Cédérom]. Dans É. Falardeau, C. Fisher, Cl. Simard \& N. Sorin (éd.), Actes du $9^{e}$ colloque de l'AIRDF. Québec: PUL.

Boutan, P. (2000). Progression et programme d'études en langue maternelle dans l'enseignement primaire à la fin du XIX siècle. Dans D. Coste et D. Véronique (éd.). La notion de progression. (pp. 71-80). Saint-Cloud: ENS Éditions.

BRISSAUD, C. (2007). Acquisition et didactique de l'orthographe du français. Dans É. Falardeau, C. Fisher, Cl. Simard \& N. SORIN (dir.). La didactique du français. Les voies actuelles de la recherche (pp. 9-26). Québec: Les Presses de l’Université Laval.

Bronckart, J.-P. \& chiss, j.-1. (1997). « Didactique », « Didactique: la didactique de la langue maternelle ». Dans Encyclopaedia Universalis France, S.A., site Universalis.fr

Bronckart, J.-P. \& SzniceR, G. (1990). Description grammaticale et principes d'une didactique de la grammaire. Le français aujourd'hui, 89, 5-16.

CHARAUDEAU, P. (2000). Grammaire du sens et de l'expression. Paris: Hachette éducation.

CHiss, J.-L. (2000). La progression: un problème typiquement didactique. Dans D. Coste et D. Véronique (éd.). La notion de progression. (pp. 67-70). Saint-Cloud: ENS Éditions.

ComEnius, J. A. (1638/2002). La grande didactique. Paris: Klincksieck. 
Coste, D. (2000). Le proche et le propre: remarques sur la notion de progression. Dans D. Coste \& d. Véronique (éd.). $L a$ notion de progression (pp. 9-20). Saint-Cloud: ENS Éditions.

Coste, D. \& Véronique, D. (2000). La notion de progression. Notions en question, $n^{\circ} 3$. Saint-Cloud: ENS Éditions.

Cycle d'orientation de l'enseignement secondaire-Genève (2001). Mémento de ponctuation à l'usage des élèves, Genève: Département de l'instruction publique.

De Pietro, J.-F. \& Schneuwly, B. (2003). Le modèle didactique du genre: un concept de l'ingénierie didactique. Les Cahiers THEODILE, 3, 27-52.

Dolz, J., Noverraz, M. \& Schneuwly, B. (2001). S'exprimer en français. Séquences didactiques pour l'oral et l'écrit. Notes méthodologiques. (Vol. IV, VII, VIII et IX). Bruxelles: De Boeck/COROME.

Dolz, J. \& Schneuwly, B. (1998). Pour un enseignement de l'oral. Initiation aux genres formels à l'école. Paris: ESF éditeur.

Dolz, J. \& Schneuwly, B. (1996). Genres et progression en expression écrite: éléments de réflexion à propos d'une expérience romande. Enjeux, 37/38, 49-75.

DUMORTIER, j.-1. (2006a). Modeste(s) proposition(s) concernant... un enseignement et un apprentissage de savoirs linguistiques qui permettent à tous les élèves de développer des compétences de communication? Enjeux, 66, 71-88.

Dumortier, j.-1. (2006b). L'observation réfléchie de la langue au premier degré du secondaire? Enjeux, 65, 65-94. garcia-debanc, cl. (1990). L'élève et la production d'écrits. Metz: CASUM.

Genevay, E. (1994). Ouvrir la grammaire. Lausanne/Montréal: L.E.P. /La Chenelière.

GREF-GRoupe de référence en français (2002). L'enseignement lapprentissage du français à l'école obligatoire. Document inédit, décembre 2002.

Grevisse, M. \& Goosse, A. (1993). Le bon usage. Paris: Duculot.

Grossman, F. \& Manesse, D. (éd., 2003). L'« observation réfléchie de la langue » à l'école. Repères, 28.

Meleuc, S. \& Fauchart, N. (1999). Didactique de la conjugaison. Le verbe « autrement ». Bertrand-Lacoste/CRDP MidiPyrénées.

MEQ-Ministère de l'Éducation du Québec. (1995). Programmes d'études. Le français: enseignement secondaire. Québec: Gouvernement du Québec. Voir le Programme d'études et l'Errata sur le site du MELS.

Nadeau, M. (1996). Le matériel scolaire et sa part de responsabilité dans les performances des écoliers en orthographe grammaticale. Revue de l'ACLA, vol. 17 (2), 65-84.

Nadeau, M. \& Fisher, C. (2006). La grammaire nouvelle. La comprendre et l'enseigner. Montréal: Gaétan Morin éditeur, La Chenelière Éducation.

Paret, M.-Ch. (2003a). La « grammaire textuelle ». Une ressource pour la compréhension et l'écriture des textes $\left(1^{\text {re }}\right.$ partie). Québec français, 128, 48-50.

Paret, M.-Ch. (2003b). La « grammaire textuelle». Une ressource pour la compréhension et l'écriture des textes $\left(2^{\mathrm{e}}\right.$ partie). Québec français, 129, 77-35.

PARET, M.-Ch. (2000). Enseigner stratégiquement la grammaire, Québec français, 119, 54-57.

PARET, M.-Ch. (1991) La syntaxe écrite des élèves du secondaire. Coll. Rapports de recherche, Montréal: Université de Montréal.

Riegel, M., Pellat, J.-C. \& Rioul, R. (1994). Grammaire méthodique du français. Paris: PUF.

Reuter, Y. (éd., 2007). Dictionnaire des concepts fondamentaux des didactiques. Bruxelles: De Boeck. 
SCHNEUWLY, B. (2007). Le « Français »: une discipline scolaire autonome, ouverte et articulée. Dans É. Falardeau, C. Fisher, Cl. Simard \& N. SORIN (dir.). La didactique du français. Les voies actuelles de la recherche (pp. 9-26). Québec: Les Presses de l'Université Laval.

Schneuwly, B. (2007). Genres écrits et oraux et forme scolaire. Enseignement et apprentissage de la langue première à l'école. In C. Boré (éd.), Construire et exploiter des corpus de genres scolaires (pp. 13-26). Namur: Presses universitaires de Namur.

SCHNEUWLY, B. (2002). «L'écriture et son apprentissage; le point de vue de la didactique. Éléments de synthèse ». Pratiques, 115-116, 237-246.

Schneuwly, B. (1995). Apprendre à écrire. Une approche socio-historique. Dans J.-Y., Boyer, J.-P. Dionne \& P. Raymond (éd.). La production de textes (pp. 73-100). Montréal : Les Éditions Logiques.

Schneuwly, B. (1994). Genres et types de discours: considérations psychologiques et ontogénétiques. Dans Y. Reuter (éd.), Les interactions lecture-écriture (pp. 155-174). Berne: Lang

Schneuwly, B. \& Dolz, J. (1997). Les genres scolaires. Des pratiques langagières aux objets d'enseignement. Repères, 15, $27-41$.

SIMARD, Cl. (1987). Réflexions sur les programmes de français. Bilan et prospectives, Québec français, 65, 66-72.

Tanguay, B. (2001). L'art de ponctuer. Montréal: Québec/Amérique.

Thyrion, F. \& Rosier, L. (2003). Français, Langue, référentiel $3^{e} / 6^{e}$ secondaire. Bruxelles: De Boeck.

Tomassone, R. (2002). Pour enseigner la grammaire II. Textes et pratiques. Paris: Delagrave.

TOMASSONNE, G. (1996). Pour enseigner la grammaire. Paris: Delagrave.

Vargas, C. (éd., 2004). Langues et études de la langue. Approches linguistiques et didactiques. Actes du colloque international de Marseille (2003). Aix-en-Provence: PUP.

\footnotetext{
${ }^{1}$ La progression des contenus d'enseignement fait généralement partie de la programmation didactique, imposée dans les programmes d'études des systèmes scolaires par les autorités politiques.
}

\footnotetext{
${ }^{3} \mathrm{Vu}$ l'absence d'uniformité d'un système scolaire francophone à un autre, nous identifions les niveaux scolaires par le nombre d'années de scolarisation depuis la première année du primaire: la $7^{\mathrm{e}}$ année correspond à la $1^{\text {re }}$ secondaire québécoise et à la $6^{\mathrm{e}}$ française.

${ }^{4}$ Nous nous situons dans une approche didactique critique de la grammaire scolaire «traditionnelle», approche dite de «nouvelle grammaire», qui ne vise pas seulement une utilisation convenable de la langue, mais un usage réfléchi, distancié, d'où la nécessité d'un enseignement réflexif des systèmes de la langue (Béguelin, 2000; Bronckart et Sznicer, 1990; Dumortier, 2006a,b; Grossman et Manesse, 2003; Nadeau et Fisher, 2006; Paret, 2000 ; Simard, 1987; Vargas, 2004).

${ }^{5}$ La grammaire désigne tout phénomène langagier régulé et normé, ce qui inclut non seulement la syntaxe, la ponctuation syntaxique et typographique, l'orthographe, mais aussi le lexique et les phénomènes textuels comme les anaphores et les règles de progression et de cohérence. Les principaux ouvrages auxquels nous nous référons pour les contenus grammaticaux à enseigner sont Béguelin, 2000; Besson, Genoud, Lipp et Nussbaum, 1979 ; Riegel, Pellat et Rioul, 1994; voir aussi Genevay, 1994; Grevisse et Goosse, 1993; Thyrion et Rosier, 2003; Tomassonne, 1996, 2002. La grammaire du sens de P. Chareaudeau (2000) est essentielle pour travailler les différentes structures qui expriment les diverses catégories sémantiques.
} 
${ }^{6}$ Par dispositifs didactiques, nous entendons un ensemble d'activités mises en œuvre avec des moyens particuliers ayant des objectifs précis, par exemple une démarche active de découverte, l'exercisation, le travail de révision et de correction de textes, le questionnement réciproque.

${ }^{7}$ COROME propose une progression à partir des genres oraux et écrits (Dolz, Noverraz \& Schneuwly, 2001).

${ }^{8}$ Une transposition didactique maladroite a fait que le concept de séquence textuelle d'Adam a été confondu avec celui de type dans le Programme de français (MEQ, 1995).

${ }^{9}$ Précisons que la justification ne fait pas partie de la typologie d'Adam. Cependant, parce qu'elle est une pratique langagière sollicitée à l'école, elle doit être enseignée. Cette conduite langagière tient de l'explication et de l'argumentation, puisque lorsqu'on justifie ses dires, on dit pourquoi on affirme telle ou telle chose (conduite explicative) et on étaie ses dires (conduite argumentative) suivant une démarche discursive qui fait appel à une rationalité commune. Il s'agit moins de convaincre le destinataire que de lui montrer que le raisonnement sur lequel s'appuie notre discours est valable.

${ }^{10}$ La tension dialectique entre «faire de la grammaire» pour mieux lire et écrire et «faire de la grammaire » pour comprendre le fonctionnement du système de la langue est représentative du courant dit de nouvelle grammaire (Bilodeau, 2005; Schneuwly, 2007; Simard, 1987). 